\title{
Sensación de inacabado y experiencias "not just right" como motivadoras de los síntomas obsesivo-compulsivos
}

\author{
Gertrudis Fornés ${ }^{1}$, M. Ángeles Ruiz-Fernández ${ }^{2}$, Amparo Belloch ${ }^{1}$ \\ ${ }^{1}$ Universidad de Valencia, Valencia, España \\ ${ }^{2}$ Universidad Nacional de Educación a Distancia, Madrid, España
}

\begin{abstract}
Resumen: La sensación de inacabado, las experiencias "not just right" y los síntomas "just right" se están postulando como variables motivadoras de síntomas obsesivos-compulsivos. En este estudio se examinaron las relaciones entre estos "nuevos" constructos y las variables síntomas, creencias disfuncionales, perfeccionismo e intolerancia a la incertidumbre asociadas al trastorno obsesivo compulsivo (TOC), en comparación con su asociación a la psicopatología emocional no-TOC. Los resultados a partir de 267 participantes que completaron auto-informes indican que las personas con riesgo de TOC puntúan más que las que no tienen riesgo en los constructos objeto de estudio. Únicamente la sensación de inacabado, las experiencias not just right y los síntomas just right fueron capaces de predecir los síntomas TOC, por encima de las creencias disfuncionales típicas del trastorno y de los síntomas no-TOC. Estos resultados indican la necesidad de tener en cuenta estas variables para mejorar la comprensión del TOC y su tratamiento.
\end{abstract}

Palabras clave: Sensación de inacabado; experiencias not just right; trastorno obesivo-compulsivo; psicopatología emocional.

Incompleteness and Not Just Right experiences as motivators of obsessive-compulsive symptoms

\begin{abstract}
The sense of incompleteness, "not just right" experiences and "just right" symptoms are considered motivational variables of obsessive-compulsive symptoms. This study examines the relationships between these "new" constructs and Obsessive-Compulsive Disorder (OCD) related variables (symptoms, dysfunctional beliefs, perfectionism, and intolerance to uncertainty), in comparison to its association with emotional non-OCD psychopathology. The results from 267 individuals who completed self-reports indicate that individuals at risk of developing OCD score higher on the main constructs of the study than those not at risk. Only the variables incompleteness, not just right experiences and just right symptoms were able to predict OCD symptoms, beyond the dysfunctional beliefs typically linked to OCD, and non-OCD symptoms. These results suggest the relevance of these variables for improving understanding $\mathrm{OCD}$ and its treatment.
\end{abstract}

Keywords: Incompleteness; not just right experiences; obsessive-compulsive disorder; emotional psychopathology.

\section{Introducción}

Los modelos cognitivo-conductuales contemporáneos resaltan la importancia de las creencias disfuncio-

Recibido: 2 junio 2015; aceptado 15 de septiembre 2015.

Correspondencia: Gertrudis Fornés, Facultad de Psicología, Universidad de Valencia, Avda. Blasco Ibáñez 21, 46010, Valencia, España. Correo-e: gertrudis.fornes@uv.es

Agradecimientos: Este trabajo forma parte de los proyectos de investigación PSI2013-44733-R financiado por el Ministerio de Economía y Competitividad del Gobierno de España, (MINECO), y PROMETEO/2013/066, financiado por la Conselleria d' Educació, Cultura i Esport de la Generalitat Valenciana. nales y la evitación del daño como factores motivadores de los síntomas obsesivo-compulsivos (p.ej., Rachman, 2002). De hecho, el énfasis en la evitación del daño es evidente en los criterios que establece el DSM-5 (American Psychiatric Association, 2013) para el diagnóstico del trastorno obsesivo-compulsivo (TOC), donde uno de los criterios que define una compulsión indica que «el objetivo de los comportamientos o actos mentales es prevenir o disminuir la ansiedad o el malestar, o evitar algún suceso o situación temida».

Las personas con TOC presentan diferentes manifestaciones sintomáticas relacionadas con la forma y el contenido de los síntomas, así como notables diferencias en 
su respuesta al tratamiento. Así, las razones por las que distintos pacientes realizan las mismas compulsiones pueden no ser las mismas: mientras una persona se lava las manos compulsivamente por miedo al contagio, otra lo hace en respuesta a la sensación de que sus manos no están como "deberían estar", a sabiendas de que no va a ocurrir nada o de que no va a contraer ninguna enfermedad que requiera un lavado excesivo o minucioso. Por ello, numerosas investigaciones indican que la etiología del TOC responde a una condición motivacionalmente heterogénea (Arnáez, García-Soriano, y Belloch, 2015; Summerfeldt, 2004), que no queda suficientemente explicada por los dos constructos que propone el enfoque cognitivo-conductual: las creencias disfuncionales que los pacientes mantienen sobre sus síntomas, y la necesidad o el impulso de evitar el riesgo o el daño que sugiere la obsesión, por pequeño o improbable que éste pueda ser.

Teniendo en cuenta estas limitaciones, en la última década se ha producido un renovado interés entre clínicos e investigadores por encontrar otras variables que permitan explicar mejor las razones que subyacen a, o motivan, los síntomas obsesivo-compulsivos. Entre ellos destacan los sentiments d'incomplétude que Pierre Janet describió en Les obsessions et la psychasténie (1903). Estos sentiments hacen referencia a la sensación interna y subjetiva de imperfección, que tortura a los pacientes pues se sienten incapaces de realizar bien sus acciones, o bien sienten que esas acciones no les producen la satisfacción que esperaban obtener con ellas a pesar de que los demás las consideren bien realizadas. Esas sensaciones estarían relacionadas de manera causal con los síntomas, y no serían por lo tanto, un mero epifenómeno de ellos. El "regreso" de los sentiments d'incompletude janetianos a la escena psicológica se produjo inicialmente de la mano de Rasmussen y Eisen (1992), que propusieron un modelo explicativo de la psicopatología obsesiva basado en tres elementos: la evaluación excesiva o anormal del riesgo, la duda patológica, y la sensación de inacabado o incompleto (incompleteness en la literatura inglesa). Desde esta perspectiva, plantearon que las compulsiones llevadas a cabo por los pacientes con TOC tendrían la finalidad de conseguir un estado de perfección y "completud".

Estas ideas han sido retomadas más recientemente por Summerfeldt (2004), que ha propuesto un modelo basado en dos dimensiones afectivo-motivacionales nucleares del TOC: la evitación del daño y la sensación de inacabado. Según esta autora, la evitación del daño se produce no solo en el TOC, pues caracteriza también a otros trastornos de ansiedad, mientras que la sensación de inacabado es única de los fenómenos obsesivo-compulsivos. Algunos estudios llevados a cabo con pobla- ción general y otros dos con pacientes con TOC, aportan datos que, en parte, apoyan la validez de este modelo (p.ej., Ecker, Gönner y Wilm, 2011). Para poner a prueba el modelo, se diseñó el cuestionario de dimensiones nucleares obsesivo compulsivas (Obsessive Compulsive Core Dimensions Questionnaire, OC-CDQ; Summerfeldt, et al., 2014), que evalúa las dos dimensiones motivacionales de síntomas obsesivo-compulsivos propuestas: sensación de inacabado y evitación del daño.

Análogas a las sensaciones de inacabado de Summerfeldt y su grupo, se habían propuesto con anterioridad otros constructos como las not just right perceptions ${ }^{l}(\mathrm{p}$. ej., Leckman, Walker, Goodman, Pauls, y Cohen, 1994) y los fenómenos sensoriales (sensory phenomena; Miguel et al., 2000), para explicar los síntomas de las personas con trastornos de tics complejos (p.ej., Leckman et al., 1994; Sarmiento, Guillén, y Sánchez, 2016). Más tarde estas percepciones y fenómenos se observaron también en pacientes con TOC, con y sin comorbilidad con trastornos de tics, hasta el punto de considerarlos como frecuentes en estos pacientes (Miguel et al., 2000), y especialmente asociados con los comportamientos compulsivos (Ferrão et al., 2012).

En el ámbito de las investigaciones cognitivo-conductuales actuales sobre el TOC, esas experiencias not just right y su estudio sistemático ha sido promovido por el grupo de Coles (Coles, Frost, Heimberg, y Rheaume, 2003), que diseñó un autoinforme específico para su evaluación, el Not Just Right Experiences Questionnaire-Revised (NJREQ-R, Coles et al., 2003). Según sus autores, la incapacidad de las personas con TOC para alcanzar su estado deseado les lleva a experimentar continuamente experiencias not just right, las cuales a su vez, generan un mayor compromiso en comportamientos compulsivos. Por tanto, la naturaleza repetitiva de las compulsiones es, en parte, resultado de las experiencias not just right. En la misma línea, y teniendo en cuenta las variables o fenómenos antes descritos, Thordarson, et al., (2004) elaboraron un instrumento, el Inventario Obsesivo Compulsivo de Vancouver (Vancouver Obsessive Compulsive Inventory; VOCI) que evalúa síntomas obsesivos compulsivos y cuenta con una subescala específica para la evaluación de síntomas just right. La diferencia

\footnotetext{
${ }^{1}$ Mantenemos la locución inglesa por dos razones: la primera, por la dificultad en apresar el significado y alcance psicológicos de la expresión en castellano mediante una locución breve; la segunda, porque es el término que se utiliza en la literatura especializada, independientemente del idioma en que esté redactado el trabajo. Su traducción al castellano refiere a un conjunto complejo de sensaciones de que hay algo que no está bien del todo, o como se supone que debería estar para decidir que «ya está correcto»o «finalizado».
} 
con el cuestionario de Coles, radica, por tanto, en que el VOCI evalúa la presencia de síntomas just right como un grupo o dimensión más de síntomas del TOC, mientras que el antes mencionado NJREQ-R evalúa si la persona ha tenido o no experiencias not just right, cuántas ha experimentado, cuáles han sido, y qué impacto han tenido en su funcionamiento.

Diversos trabajos han estudiado hasta el momento los dos constructos señalados, sensaciones de inacabado y experiencias not just right. Algunos analizan las asociaciones que se establecen entre la sensación de inacabado y fenómenos análogos con variables más generales, como el perfeccionismo (Coles et al., 2003; Moretz y McKay, 2009), que aluden tanto a creencias como a características de personalidad. Otras investigaciones centraron su atención en el estudio de la relación de ambos constructos con los subtipos de TOC (Cougle et al., 2013). Por ejemplo, Ecker y Gönner (2008), encontraron en una muestra de pacientes con TOC que los síntomas de orden y comprobación (y no los de contaminación/ lavado y las obsesiones) se asociaban con sensación de inacabado. Sin embargo, no tenemos constancia de trabajos que estudien la relación entre los dos constructos mencionados con variables como la intolerancia a la incertidumbre y las creencias disfuncionales asociadas al TOC.

Algunos estudios llevados a cabo tanto en muestras clínicas como no clínicas revelan una elevada asociación entre la sensación de inacabado (y fenómenos similares surgidos a continuación), y también la evitación del daño, con sintomatología TOC (Belloch et al., 2016; Coles et al., 2003, 2005; Ecker y Gönner, 2008; Ferrão et al., 2012; Taylor et al., 2014), resultados que confirman la importancia de estas dimensiones en el TOC. Sin embargo, otros estudios ponen en entredicho la especificidad de estos fenómenos para el TOC. Así, Fergus (2013) encontró en una muestra de personas de la población general que las experiencias not just right eran relevantes para un amplio grupo de trastornos emocionales como la depresión, la ansiedad generalizada, o la ansiedad social. Por su parte, un reciente meta-análisis de Taylor et al., (2014) revela asociaciones entre las experiencias not just right y la sensación de inacabado con síntomas de depresión y ansiedad general. Estos resultados podrían indicar que estos "nuevos" constructos tienen carácter transdiagnóstico y son, por tanto, compartidos por trastornos emocionales diferentes. De ser así, permitiría entender mejor la elevada comorbilidad que existe entre estos trastornos, o incluso configurar, como dicen Kloosterman, Summerfeld, Parker y Holden (2013), un endofenotipo compartido por diferentes trastornos en los que esté afectado el control inhibitorio, es decir, una característica que posiblemente tiene un origen genético y que podría constituir un riesgo de padecer un trastorno, sin considerarse un síntoma en sí mismo del trastorno. Parece pues necesario profundizar en las investigaciones, para dilucidar el rol de estas experiencias en el TOC y en otros trastornos diferentes (Barajas, 2015).

En consecuencia con lo mencionado hasta aquí, los objetivos de este trabajo son: (1) examinar de forma específica la relación entre los "nuevos" constructos sensación de inacabado y experiencias not just right (tanto a nivel de síntomas como de experiencias en sí), (2) analizar la asociación de ambos constructos con sintomatología emocional TOC y no-TOC, y con las variables típicas de la orientación cognitivo-conductual del TOC (i.e., evitación del daño y creencias disfuncionales), (3) investigar la posible dimensionalidad de la sensación de inacabado y las experiencias not just right (esto es, que se sitúen en un continuo de intensidad, discurriendo desde la normalidad a la psicopatología), y (4) estudiar la capacidad de estos constructos para predecir las dimensiones de síntomas obsesivo-compulsivos, por encima del peso explicativo que puedan tener otras variables como las creencias disfuncionales, el perfeccionismo, la intolerancia a la incertidumbre, la tendencia a la preocupación patológica (worry), y los síntomas ansiosos y depresivos que, a menudo, acompañan al TOC.

Sobre la base de la literatura revisada y a los trabajos analizados, se plantean las siguientes hipótesis: (1) Especificidad: las sensación de inacabado, las experiencias not just right, y los síntomas just right se asociarán de manera significativa con variables conceptualmente vinculadas con el TOC: intolerancia a la incertidumbre, perfeccionismo y creencias disfuncionales. Asimismo, su asociación con síntomas TOC será mayor que la vinculada con psicopatología emocional no-TOC. (2) Dimensionalidad: las personas vulnerables o en riesgo de padecer TOC, comparadas con las personas sin riesgo, presentarán puntuaciones significativamente más elevadas en medidas de los tres constructos objeto de estudio. (3) Capacidad predictiva: La sensación de inacabado, las experiencias not just right y los síntomas just right emergerán como predictores significativos de las diferentes dimensiones de síntomas obsesivo-compulsivos, una vez controlada la potencial capacidad predictiva de otras variables y síntomas vinculados con el TOC.

\section{Método}

\section{Participantes}

La muestra estuvo constituida por un total de 267 personas, de las cuales el 74.5\% eran mujeres. La edad media de los participantes era de 27.29 años $(D T=11.79$; 
rango $=18-60$ años), y la mayoría de ellos informó estar soltero $(73.4 \%)$ y tener estudios universitarios $(80.9 \%)$. Asimismo, se preguntó a los participantes por el nivel socio-económico al que considera que pertenece mediante alternativas de respuesta (bajo, medio-bajo, medio, medio-alto, alto). El 67.4\% de los participantes seleccionó un nivel socio-económico medio.

\section{Instrumentos de evaluación}

Cuestionario de Dimensiones Nucleares Obsesivo-Compulsivas (Obsessive-Compulsive Core Dimensions Questionnaire, OC-CDQ; Summerfeldt, Kloosterman, Antony y Swinson, 2014). Auto-informe que evalúa dos dimensiones motivacionales del TOC: evitación del daño y sensación de inacabado. Está compuesto por 20 ítems con una escala de respuesta tipo Likert de 5 puntos que oscilan desde 0 (nunca) a 4 (siempre). La validación en población española fue llevada a cabo por Carrasco y Belloch (2013) y sus dimensiones obtuvieron unos excelentes índices de consistencia interna $(\alpha=0.92)$. En el presente estudio, estos índices fueron satisfactorios: $\alpha=$ 0.89 para la subescala de evitación del daño y $\alpha=0.88$ para la de sensación de inacabado.

Cuestionario de Experiencias no del Todo Correctas-Versión Revisada (Not Just Right Experiences Questionnaire-Revised, NJREQ-R; Coles, et al., 2005. Versión en español de Carrasco y Belloch, 2013). Auto-informe compuesto por 19 ítems desarrollado para medir el número de experiencias not just right, y la gravedad de la experiencia más reciente. Los primeros 10 ítems corresponden a una lista de experiencias not just right y mediante una escala de respuesta dicotómica la persona debe responder si, durante el último mes, ha experimentado o no cada una de las experiencias de la lista. Los ítems 11 y 12 hacen referencia a qué experiencia de la lista le ha ocurrido más recientemente, y cuándo fue la última vez que le ocurrió (de 1 = en las últimas horas a 4 $=$ en el último mes). En los siguientes 7 ítems (rango de respuesta entre 1 = nada a 7 = extremadamente), la persona tiene que valorar la experiencia más reciente (anotada en el ítem 11) en cuanto a frecuencia con que le ocurre, intensidad con que la experimenta, malestar que le produce cuando la experimenta, malestar posterior, rumiación y/o dificultad de eliminarla, impulso o necesidad de hacer algo, y responsabilidad que siente en relación con esa experiencia not just right. La suma de estos ítems se utiliza como valoración de gravedad de la experiencia not just right evaluada. Carrasco y Belloch (2013) encontraron que la fiabilidad de la lista de experiencias not just right fue $\alpha=0.63$, mientras que la escala de gravedad mostró un valor de $\alpha=0.85$. En el presente estudio, la consisten- cia interna para los primeros 10 ítems fue $\alpha=0.59$, y para la escala de gravedad fue $\alpha=0.86$.

Inventario Obsesivo-Compulsivo de Vancouver (Vancouver Obsessive-Compulsive Inventory, VOCI; Thordarson, et al., 2004. Validación en Español: Nogueira et al., 2009). Este cuestionario evalúa síntomas obsesivo-compulsivos a través de 55 ítems, con una escala de respuesta de cinco puntos $(0=$ en absoluto, $4=$ muchísimo $)$. Los ítems se agrupan en 6 escalas: contaminación (12 ítems), comprobación (6 ítems), obsesiones (12 ítems), acumulación ( 7 ítems), just right (12 ítems) e indecisión (6 ítems). El grupo de Nogueira encontró que los coeficientes de fiabilidad de las subescalas fueron entre aceptables y buenos en dos tipos de muestras (estudiantes: $\alpha=0.78-0.92$ y no-estudiantes: $\alpha=0.81-0.90$ ). Para los objetivos de este trabajo solo se empleó la escala "just right", de la que se obtuvo una consistencia interna satisfactoria $(\alpha=0.84)$.

Escala de Intolerancia a la Incertidumbre (Intolerance of Uncertainty Scale, IUS; Freeston, Rhéaume, Letarte, Dugas, y Ladouceur, 1994). Consta de 27 ítem y su escala de respuesta oscila de 1 (no es nada típico en mí, no me caracteriza nada) a 5 (es totalmente típico en mí, siempre me sucede así). Puede obtenerse una puntuación total o dos factores: efectos negativos de la incertidumbre y necesidad de control. La versión empleada en este estudio es la traducida y adaptada por Carrasco (2005), quien encontró un valor de consistencia interna excelente para la escala total $(\alpha=0.93)$, idéntico al encontrado en la muestra de este trabajo.

Escala Multidimensional de Perfeccionismo de Frost (Frost Multidimensional Perfectionism Scale, FMPS; Frost et al., 1990). Evalúa el perfeccionismo a través de 35 ítems con escala de respuesta entre 1 (completamente en desacuerdo) y 5 (completamente de acuerdo). Los ítems están agrupados en seis dimensiones: exigencias personales, preocupación por los errores, dudas sobre acciones, expectativas paternas, críticas paternas y organización. La validación del instrumento en población española fue llevada a cabo por Carrasco, Belloch y Perpiñá (2010), quienes hallaron un valor de $\alpha=0.93$ para la escala total. En el presente estudio, el coeficiente de consistencia interna para la escala total fue excelente: $\alpha=0.92$, mientras que el rango de valores para las subescalas fue satisfactorio de $\alpha=0.72$ a $\alpha=0.88$.

Inventario de Creencias Obsesivas-Revisado (ICO-R; Belloch, Morillo, Luciano, García-Soriano, Cabedo, y Carrió, 2010). Autoinforme diseñado para evaluar las creencias disfuncionales teóricamente implicadas en el desarrollo y/o mantenimiento del TOC. Está compuesto por 50 ítems con una escala de respuesta tipo Likert de 7 puntos $(1=$ completamente en desacuerdo a $7=$ completamente de acuerdo). Los ítems se agrupan en 8 escalas: responsa- 
bilidad excesiva, importancia de los pensamientos, fusión pensamiento acción (FPA)-probabilidad, FPA-moral, importancia de controlar los pensamientos, sobreestimación del peligro, intolerancia a la incertidumbre y perfeccionismo. La fiabilidad de la escala total de la validación española del instrumento fue $\alpha=0.94$, y para las subescalas los valores de $\alpha$ se situaron entre 0.69 y 0.87 . La consistencia interna mostrada por la puntuación total de la escala en este estudio fue excelente $(\alpha=0.94)$, y el rango para las subescalas se situó entre $\alpha=0.71$ y $\alpha=0.82$.

Inventario Obsesivo Compulsivo-Revisado (Obsessive Compulsive Inventory-Revised, OCI-R; Foa et al., 2002. Validación para su uso en muestras no-clínicas y clínicas de Belloch et al., 2013). Autoinforme de 18 ítems que mide el malestar asociado a los síntomas obsesivos-compulsivos. Su escala de respuesta oscila entre 0 (ausencia de malestar) y 4 (muchísimo malestar). Se puede obtener una puntuación total de la escala, o puntuaciones diferenciadas para cada una de las siguientes subescalas de síntomas obsesivo-compulsivos: lavado, comprobación, obsesiones, orden, acumulación y neutralización. En el presente estudio se obtuvo un alfa de Cronbach para la escala total de 0.88 , mientras que para las dimensiones de síntomas los valores se situaron en un rango entre $\alpha=0.56$ y $\alpha=0.81$.

Escalas de depresión, ansiedad y estrés (Depression, Anxiety and Stress Scales, DASS-21; Lovibond y Lovibond, 1995). Instrumento diseñado para evaluar la depresión, la ansiedad y el estrés en adultos. Consta de 21 ítems con 4 alternativas de respuesta $(0=$ nunca, no me pasó, a 3 = casi todo el tiempo). Se empleó la validación española de Bados, Solanas y Andrés (2005), quienes encontraron índices de fiabilidad elevados para los tres conjuntos de síntomas (depresión: $\alpha=0.92$, ansiedad: $\alpha$ $=0.84$ y estrés: $\alpha=0.91)$. Los valores de consistencia interna obtenidos con la presente muestra fueron: ansiedad: $\alpha=0.70$; estrés: $\alpha=0.76$ ); depresión: $\alpha=0.80$.

Inventario de Preocupación de Pensilvania (Penn State Worry Questionnaire, PSWQ; Meyer, Miller, Metzger y Borkovec, 1990. Validación española: Sandín, Chorot, Valiente, y Lostao, 2009). Autoinforme que evalúa la tendencia a preocuparse por diferentes aspectos de la vida cotidiana. Consta de 16 ítems, que se puntúan según una escala tipo Likert de 1 (nada típico en mí) a 5 (muy típico en mí). La consistencia interna obtenida en este estudio fue la misma que la hallada por los autores de la validación en población española del instrumento $(\alpha=0.90)$.

\section{Procedimiento}

Este estudio contó con la colaboración voluntaria de estudiantes de grado de Psicología procedentes de la
Universidad de Valencia y de la Universidad Nacional de Educación a Distancia (UNED). El procedimiento de recogida de los datos fue diferente en los dos grupos. En el caso de los estudiantes de la Universidad de Valencia, su participación consistió en la asistencia a un seminario de investigación, donde se explicaron los aspectos teóricos en los que se enmarcaba la investigación, y el sentido y la importancia de su colaboración. En la misma sesión, se describieron los instrumentos que componían el protocolo de evaluación y se entrenó a los asistentes en la forma de administrarlos. A continuación, se les pidió que administraran la batería de cuestionarios, de forma anónima e individualizada, a cuatro familiares y/o amigos. En consecuencia, son los datos recabados por los estudiantes, y no los suyos, los que se incluyen en el estudio. Por su parte, la colaboración de los estudiantes de la UNED consistía en cumplimentar el mismo protocolo de cuestionarios mencionado más arriba, pero mediante el empleo del programa SurveyMonkey, software diseñado para la creación de encuestas y cuestionarios online. Así, los estudiantes recibieron, a través de sus correos electrónicos, un link que les permitía responder la totalidad de los instrumentos.

Para ambos grupos el orden de administración de los instrumentos se balanceó aleatoriamente para configurar los protocolos. Todos los participantes de este estudio firmaron un consentimiento informado en el que aceptaban participar de forma voluntaria y daban su conformidad para que los datos derivados de la colaboración pudieran ser utilizados en futuras investigaciones, salvaguardando la identidad de los participantes, Este estudio, que forma parte de un proyecto de investigación más amplio, fue aprobado por el Comité Ético de la Universidad de Valencia.

\section{Análisis estadísticos}

La totalidad de los análisis llevados a cabo en este trabajo se realizaron con el programa SPSS v.20 (SPSS, Chicago, IL, USA). En primer lugar, se calcularon los estadísticos descriptivos para los tres constructos centrales del estudio. Por otro lado, para analizar las asociaciones se realizaron correlaciones bivariadas (Pearson), y las diferencias entre dichas correlaciones se determinaron mediante el cálculo del estadístico $z$ de Fisher. Para valorar el tamaño de las correlaciones, se adoptaron los criterios de Cohen (1998): pequeño: $r=0.10$ a 0.29 ; medio/moderado: $r=0.30$ a 0.49 ; grande: $r=\geq 0.50$ ).

Las diferencias de medias entre dos grupos se analizaron mediante el estadístico $t$ de Student para grupos independientes y con el estadístico $d$ de Cohen para el cálculo del tamaño del efecto. Finalmente, para estudiar 
el poder predictivo de la sensación de inacabado, las experiencias not just right y los síntomas just right en la explicación de los síntomas obsesivo-compulsivos, se efectuaron una serie de regresiones lineales múltiples por bloques. En cada uno de los análisis se empleó como variable dependiente cada una de las dimensiones de síntomas TOC, y se controló el potencial peso que la sintomatología no-TOC y las creencias disfuncionales pudieran tener en la mencionada predicción.

\section{Resultados}

Datos preliminares sobre la sensación de inacabado, las experiencias not just right, y los sintomas just right

Los resultados de los estadísticos descriptivos muestran que estos fenómenos ocurren en la población general (Tabla 1). La puntuación media de la sensación de inacabado fue mayor que la alcanzada para la dimensión de evitación del daño $(t=13,67 ; p<0.001)$. Así, mientras que para la primera variable, la media obtenida se corresponde con el indicador "siempre pienso, siento, o actúo así" en la escala de respuesta, para la segunda el valor de la media se ajusta a "algunas veces" en la escala de respuesta. Además, cabe destacar que el $96.3 \%$ de los participantes puntuó por encima de 1 en evitación del daño, mientras que la totalidad de la muestra obtuvo una puntuación por encima de 2 en sensación de inacabado.
Respecto a las experiencias not just right, una gran mayoría de los participantes en este estudio, refirió haber tenido al menos una de las 10 experiencias que se incluyen en el cuestionario. Entre quienes las experimentaron, la intensidad media se corresponde con un indicador que oscila entre "un poco" y "moderadamente" en la escala de respuesta del cuestionario. En cuanto a los síntomas just right (evaluados con el VOCI), de nuevo la mayoría de la muestra obtuvo una puntuación por encima del valor 1que en la escala de respuesta se traduce en "algo".

\section{Dimensionalidad de la sensación de inacabado, las experiencias not just right y los sintomas just right}

Para examinar la dimensionalidad de las variables objeto de estudio, analizamos las diferencias entre las personas con alto y bajo riesgo de presentar TOC. Para ello, la muestra se dividió en dos grupos tomando como criterio de corte una puntuación total en el OCI-R $\geq 14$. Esta puntuación coincide con la media obtenida en este instrumento por los participantes en el estudio, y en la validación española del OCI-R (Belloch et al., 2013) la puntuación total de 14 se corresponde con un valor predictivo positivo de $71.7 \%$, un valor predictivo negativo de $79.4 \%$, y un índice Youden de 0.49 . Teniendo en cuenta el mencionado punto de corte, un total de 105 participantes se pueden considerar como vulnerables a presen-

Tabla 1. Estadísticos de tendencia central de las medidas de sensación de inacabado, experiencias not just right y síntomas just right

\begin{tabular}{|c|c|c|c|c|c|c|}
\hline Variables & $M(D T)$ & Mín & Máx. & Asimetría & $\%>1$ & $n$ \\
\hline \multicolumn{7}{|l|}{ OC-CDQ: } \\
\hline Evitación del daño & $10.11(6.69)$ & 0 & 34 & 0.80 & 96.3 & 267 \\
\hline Sensación de inacabado & $14.60(6.87)$ & 2 & 36 & 0.49 & 100 & 267 \\
\hline \multicolumn{7}{|l|}{ NJREQ-R: } \\
\hline Número de experiencias not just right & $3.08(2.03)$ & 0 & 10 & 0.54 & 91 & 267 \\
\hline Frecuencia de la más reciente & $5.40(1.25)$ & 2 & 7 & -0.64 & 100 & 243 \\
\hline Intensidad de la más reciente & $3.08(1.35)$ & 1 & 7 & 0.21 & 100 & 243 \\
\hline Malestar en el momento (más reciente) & $2.93(1.46)$ & 1 & 7 & 0.31 & 100 & 243 \\
\hline Malestar a largo plazo (más reciente) & $2.03(1.44)$ & 0 & 7 & 1.24 & 99.6 & 243 \\
\hline Dificultad de eliminar la más reciente & $1.75(1.27)$ & 0 & 7 & 1.78 & 99.6 & 243 \\
\hline Necesidad de hacer algo (más reciente) & $2.76(1.65)$ & 0 & 7 & 0.69 & 99.6 & 243 \\
\hline Responsabilidad (más reciente) & $2.98(1.87)$ & 0 & 7 & 0.48 & 99.6 & 243 \\
\hline Gravedad de la experiencia NJR más reciente & $20.93(7.21)$ & 9 & 48 & 0.86 & 100 & 256 \\
\hline VOCI-Síntomas just right & $7.13(6.35)$ & 0 & 46 & 1.88 & 94.8 & 267 \\
\hline
\end{tabular}

Nota. $\%>1=$ porcentaje de personas que puntúan por encima de 1; Max - Min = valores máximo y mínimo; NJREQ-R: Not Just Right Experiences Questionnaire-Revised; OC-CDQ: Obsessive Compulsive Core Dimension Questionnaire; VOCI: Vancouver Obsessive Compulsive Inventory. 
tar síntomas de TOC, y 162 no vulnerables. Los resultados de las comparaciones entre los dos grupos establecidos se muestran en la Tabla 2. Como puede constatarse, los dos grupos se diferenciaron con claridad en todas las medidas (tamaños del efecto grandes), obteniendo las personas con riesgo de presentar síntomas obsesivo-compulsivos puntuaciones más elevadas en todos los casos.

Tabla 2. Diferencias entre personas en riesgo de TOC $(n=105)$ y personas $\sin$ riesgo $(n=162)$ en sensación de inacabado, evitación del daño, experiencias not just right, y síntomas just right

\begin{tabular}{lcccc}
\hline \multicolumn{1}{c}{ Variables } & Sin riesgo de TOC & Subclínicos TOC & $t(265)$ & \multicolumn{1}{c}{$d$ de Cohen } \\
\hline OC-CDQ-Sensación de inacabado & $12.06(5.42)$ & $18.51(7.05)$ & $7.967^{*}$ & $6.505^{*}$ \\
OC-CDQ-Evitación del daño & $8.12(5.59)$ & $13.19(7.09)$ & $5.936^{*}$ & 0.799 \\
NJREQ-R-Número & $2.52(1.91)$ & $3.94(1.91)$ & $6.488^{*}$ & 0.729 \\
NJREQ-R-Gravedad & $17.31(7.50)$ & $23.71(8.04)$ & $7.577^{*}$ & 0.797 \\
VOCI-síntomas justright & $4.75(3.93)$ & $10.80(7.53)$ & 0.712 \\
\hline
\end{tabular}

Nota. Los datos se expresan como Media (DT). OC-CDQ: Obsessive Compulsive Core Dimension Questionnaire; NJREQ-R: Not Just Right Experiences Questionnaire-Revised; VOCI: Vancouver Obsessive Compulsive Inventory. ${ }^{*} p<0.0001$.

Relaciones entre sensación de inacabado, experiencias not just right, sintomas just right y creencias asociadas al TOC

Con la finalidad de analizar las asociaciones entre la sensación de inacabado, el número de experiencias not just right, la gravedad de la experiencia not just right más reciente y los síntomas just right, se realizó un análisis de correlación bivariada entre los tres instrumentos que evalúan estas variables (Tabla 3). Los resultados indicaron correlaciones entre moderadas y grandes entre las tres medidas, excepto la asociación entre número de experiencias not just right y evitación del daño, cuyo tamaño fue menor.

El tamaño de la correlación entre las dos subescalas del OC-CDQ, evitación del daño y sensación de inacabado fue grande. Además, el cálculo de las diferencias entre correlaciones, reveló que la asociación entre la sensación de inacabado y síntomas just right (medidos con el cuestionario VOCI) fue mayor que la obtenida entre sensación de inacabado y evitación del daño $(z=3.48 ; p$ $<0.05$ ). Por su parte, la correlación entre el número de experiencias not just right y la gravedad de la experiencia not just right más reciente fue moderada. Asimismo, no se encontraron diferencias significativas entre las correlaciones de ambas escalas con el resto de las medidas. En cuanto a los síntomas VOCI-just right, se asociaron de forma más importante con la sensación de inacabado que con el número de experiencias not just right $(z=$ 5.83; $p<0.05)$ y que con la gravedad de la experiencia not just right más reciente $(z=4.73 ; p<0.05)$.

En cuanto a las relaciones entre los indicadores objeto de estudio y las medidas que se asocian al TOC, los resultados obtenidos indican que la intolerancia a la incertidumbre, los rasgos de perfeccionismo y las creencias disfuncionales asociadas al TOC están relacionadas de forma moderada con la sensación de INC, la gravedad de la experiencia not just right más reciente, y los síntomas just right. Por su parte, la evitación del daño se asocia más a creencias disfuncionales características del TOC $(z=4.34 ; p<0.05)$ y a intolerancia a la incertidumbre $(z=3 ; p<0.05)$ que al perfeccionismo. Lo mismo ocurre con la sensación de inacabado, pues mantiene asociaciones más altas con creencias disfuncionales $(z=2.91 ; p<0.05)$ y con intolerancia a la incertidumbre $(z=2.34 ; p<0.05)$, que con el rasgo de perfeccionismo (FMPS). Por lo que se refiere al número de experiencias not just right, gravedad de la experiencia not just right más reciente y síntomas just ri$g h t$, no hay diferencias en las correlaciones entre estas variables y las variables TOC-relacionadas, es decir, se asocian en igual proporción con intolerancia a la incertidumbre, perfeccionismo y creencias disfuncionales.

Relaciones entre la sensación de inacabado, las experiencias not just right, los sintomas just right, la psicopatología emocional no-TOC, y las dimensiones de sintomas TOC

En primer lugar, se realizó un análisis de correlación cuyos resultados indicaron que la evitación del daño, la sensación de inacabado, el número de experiencias not just right, la gravedad de la experiencia not just right más reciente y los síntomas just right, mantenían asociaciones con las medidas de psicopatología emocional no-TOC y con los síntomas TOC (OCI-R) empleados en este estudio. Los resultados se presentan en la Tabla 4. En concre- 
Tabla 3. Correlaciones bivariadas entre medidas de sensación de inacabado y experiencias not just right con creencias disfuncionales, intolerancia a la incertidumbre y perfeccionismo $(N=267)$

\begin{tabular}{|c|c|c|c|c|c|c|c|}
\hline & \multicolumn{2}{|c|}{ OC-CDQ } & \multicolumn{2}{|c|}{ NJREQ-R } & \multirow{2}{*}{ IUS } & \multirow{2}{*}{ FMPS } & \multirow{2}{*}{ ICO-r } \\
\hline & Evitación daño & Inacabado & Número & Gravedad & & & \\
\hline OC-CDQ-Evitación daño & -- & & & & .519 & .304 & .599 \\
\hline OC-CDQ-Inacabado & .688 & -- & & & .613 & .470 & .643 \\
\hline NJREQ-R-Número & .278 & .330 & -- & & .327 & .284 & .335 \\
\hline NJREQ-R-Gravedad & .380 & .407 & .452 & -- & .475 & .338 & .473 \\
\hline VOCI- just right & .529 & .712 & .366 & .446 & .603 & .539 & .574 \\
\hline
\end{tabular}

Nota. Todas las correlaciones $p \leq 0.001$. OC-CDQ: Obsessive Compulsive Core Dimension Questionnaire; NJREQ-R: Not Just Right Experiences Questionnaire-Revised; VOCI: Vancouver Obsessive Compulsive Inventory; IUS: Intolerance Uncertainty Scale; FMPS: Frost Multidimensional Perfectionism Scale; ICO-r: Inventario de Creencias Obsesivas-Revisado.

to, la evitación del daño se relacionó de forma moderada con todos los indicadores de psicopatología, mientras que la sensación de inacabado se asoció más con síntomas obsesivos compulsivos y con la tendencia a preocuparse (PSWQ), que con síntomas de estrés, ansiedad y depresión (DASS-21) (valores $z$ en la Tabla 4).

El número de experiencias not just right mantuvo una relación significativamente mayor con la sintomatología obsesivo-compulsiva que con el estrés, la depresión y las preocupaciones (ver los valores $z$ en la Tabla 4). Sin embargo, la gravedad de la experiencia not just right más reciente se asoció significativamente más con los síntomas obsesivos compulsivos, el estrés, la depresión y las preocupaciones, que con los síntomas de ansiedad. Por su parte, los síntomas just right (VOCI), se asociaron de forma más potente con los síntomas obsesivo-compulsivos que con el estrés, la ansiedad, la depresión y las preocupaciones (todos los valores $z$ en la Tabla 4).

Tabla 4. Correlaciones bivariadas y diferencia de correlaciones para las medidas de sensación de inacabado, experiencias not just right, síntomas just right y medidas de psicopatología emocional $(N=267)$

\begin{tabular}{|c|c|c|c|c|c|}
\hline \multirow{2}{*}{ Escalas } & \multicolumn{2}{|c|}{ OC-CDQ } & \multicolumn{2}{|c|}{ NJREQ } & \multirow{2}{*}{$\frac{\text { VOCI }}{\text { Just right }}$} \\
\hline & Evitación daño & Inacabado & Número & Gravedad & \\
\hline OCI-R-Lavado & $.413 * * *$ & $.386^{* * *}$ & $.303 * * *$ & $.296^{* * *}$ & $.459 * * *$ \\
\hline OCI-R-Comprobación & $.434 * * *$ & $.446 * * *$ & $.375^{* * *}$ & $.400 * * *$ & $.478 * * *$ \\
\hline OCI-R-Obsesiones & $.465 * * *$ & $.391 * * *$ & $.300 * * *$ & $.369 * * *$ & $.385 * * *$ \\
\hline OCI-R-Acumulación & $.330 * * *$ & $.414 * * *$ & $.294 * * *$ & $.343 * * *$ & $.484 * * *$ \\
\hline OCI-R-Neutralización & $.267 * * *$ & $.296 * * *$ & $.243 * * *$ & $.236^{* * *}$ & $.426 * * *$ \\
\hline OCI-R-Orden & $.309 * * *$ & $.542 * * *$ & $.344 * * *$ & $.320 * * *$ & $.501 * * *$ \\
\hline OCI-R-Total & $.523 * * *$ & $.588 * * *$ & $.439 * * *$ & $.474 * * *$ & $.635 * * *$ \\
\hline DASS-Estrés & $.438 * * *$ & $.382 * * *$ & $.284 * * *$ & $.425^{* * *}$ & $.346 * * *$ \\
\hline DASS-Ansiedad & $.402 * * *$ & $.271 * * *$ & $.308 * * *$ & $.301 * * *$ & $.274 * * *$ \\
\hline DASS-Depresión & $.405^{* * *}$ & $.267 * * *$ & $.218 * * *$ & $.354 * * *$ & $.278 * * *$ \\
\hline PSWQ & $.434 * * *$ & $.544 * * *$ & $.180 * *$ & $.428 * * *$ & $.439 * * *$ \\
\hline Diferencia entre correlaciones $(z)$ : OCI-R total vs. DASS-estrés & 1.27 & $3.13 * * *$ & $2.06^{*}$ & .71 & $4.47 * * *$ \\
\hline Diferencia entre correlaciones $(z)$ : OCI-R total vs. DASS-ansiedad & 1.77 & $4.56 * * *$ & 1.75 & $2.35^{*}$ & $5.38 * * *$ \\
\hline Diferencia entre correlaciones $(z)$ : OCI-R total vs. DASS-depresión & 1.73 & $4.61 * * *$ & $2.87 * *$ & 1.67 & $5.33 * * *$ \\
\hline Diferencia entre correlaciones $(z)$ : OCI-R total vs. PSWQ & 1.33 & .74 & $3.32 * * *$ & .66 & $3.2 * * *$ \\
\hline
\end{tabular}

Nota. OCI-R: Obsessive Compulsive Inventory-Revised; DASS: Depression, Anxiety and Stress Scales; PSWQ: Penn State Worry Questionnaire; OC-CDQ: Obsessive Compulsive Core Dimension Questionnaire; NJREQ-R: Not Just Right Experiences Questionnaire-Revised; VOCI: Vancouver Obsessive Compulsive Inventory ${ }^{*} p<0.05 ; * p<0.01 ; * * p<0.001$. 
Por último, cabe destacar que las cuatro variables objeto de estudio, sensación de inacabado, número de experiencias not just right, gravedad de la experiencia not just right más reciente, y los síntomas just right, mantuvieron un patrón de asociaciones moderadas con todas las dimensiones de síntomas obsesivo-compulsivos evaluados con el OCI-R (ver Tabla 4).

\section{Capacidad predictiva de las sensaciones de inacabado $y$ de las experiencias y sintomas not just right en relación a los sintomas obsesivo-compulsivos}

Dado que las variables objeto de estudio se postulan como motivadoras de los síntomas característicos del TOC, realizamos 6 análisis de regresión múltiple por bloques en cada uno de los cuales la variable dependiente fue la puntuación en una de las seis subescalas del OCI-R. Se controló el peso que pudieran tener los síntomas no-TOC y las creencias disfuncionales en la capacidad explicativa de cada una de las modalidades de síntomas obsesivo-compulsivos. En el primer bloque de cada análisis se introdujeron las puntuaciones en PSWQ y DASS-21. En el segundo bloque, se introdujeron las puntuaciones totales en las creencias (cuestionario ICO-R), intolerancia a la incertidumbre (cuestionario IUS) y perfeccionismo (cuestionario FMPS). En el tercer bloque, se introdujeron las variables del estudio: evitación del daño, sensación de inacabado, NJREQ-R-número, NJREQ-R-gravedad, y VOCI-síntomas just right. Los resultados se muestran en la Tabla 5. Únicamente se incluyen los predictores que resultaron ser significativos en cada uno de los análisis.

Los resultados muestran que la evitación del daño emergió como predictor de los síntomas de lavado, comprobación y obsesiones, mientras que la sensación de inacabado predijo los síntomas de comprobación y orden. Por su parte, los síntomas just right predijeron todas las dimensiones de síntomas obsesivo-compulsivos, excepto el referido a obsesiones "puras". En cuanto a las experiencias not just right, el número de experiencias sólo predijo los síntomas de orden, mientras que la gravedad de la experiencia not just right más reciente no predijo ninguna de las modalidades de síntomas obsesivo-compulsivos. Otras variables predictoras fueron la ansiedad (para los síntomas de orden), y la intolerancia a la incertidumbre (para los síntomas de acumulación). Es importante señalar que las creencias disfuncionales asociadas a los pensamientos no explicaron ninguna de las seis modalidades de síntomas.

\section{Discusión}

El objetivo principal de este trabajo ha sido contribuir al conocimiento de tres "nuevas" variables propues- tas como relevantes en la explicación motivacional del TOC: sensación de inacabado, experiencias not just right y síntomas just right. Hasta donde sabemos, este es el primer estudio llevado a cabo en el contexto español que examina estos constructos de forma conjunta, así como las relaciones que presentan con medidas de psicopatología emocional TOC y no-TOC.

En primer lugar, cabe mencionar que la sensación de inacabado, las experiencias not just right y los síntomas just right son habituales, pues fueron experimentados por un alto porcentaje de la población general, resultado que concuerda con estudios previos en los que se emplearon muestras similares a la de este trabajo (Coles et al., 2003; Fergus, 2013).

Por otra parte, los análisis de diferencias entre grupos en las variables objeto de estudio, revelaron puntuaciones significativamente más elevadas en el grupo con riesgo de padecer TOC, en comparación con el grupo sin riesgo de desarrollar este trastorno. Este dato sugiere que la presencia de estas experiencias de forma más intensa (y frecuente, en el caso de las experiencias not just right), podría considerarse como un factor/marcador de vulnerabilidad para desarrollar síntomas obsesivo-compulsivos. En suma, el hecho de que las experiencias objeto de estudio se den en personas de la población general, y que existan diferencias en su frecuencia entre personas con y sin riesgo de TOC, avala la hipótesis de la dimensionalidad, según la cual estos fenómenos se sitúan en un continuo de intensidad (frecuencia) que transita desde la normalidad a la psicopatología.

La dimensionalidad de los síntomas obsesivo-compulsivos es un hecho ampliamente constatado desde el estudio pionero de Rachman y de Silva (1978), quienes encontraron que los pensamientos intrusos de contenido obsesivo son comunes en la población general no clínica, y se parecen a las obsesiones tanto en la forma en que se experimentan, como en su contenido. Las diferencias entre esos pensamientos intrusos y las obsesiones radican en la mayor frecuencia, interferencia, y consecuencias que generan en la vida de las personas las obsesiones clínicamente significativas. En este sentido, las experiencias not just right y la sensación de inacabado serían asimilables a las obsesiones, y quizá pueden conceptualizarse incluso como una forma de presentación clínica de la patología obsesiva, que puede coexistir con otras dimensiones o modalidades de síntomas. La propuesta de Coles y su grupo (Coles et al., 2003, 2005) sobre la consideración de las experiencias not just right como análogas a las obsesiones encuentra un cierto apoyo atendiendo a nuestros datos.

No obstante, hay que señalar que, a pesar de que las obsesiones comparten características con las experien- 
Tabla 5. Predictores de las dimensiones de síntomas obsesivo-compulsivos

\begin{tabular}{|c|c|c|c|c|c|c|c|}
\hline Variables & $R^{2}$ Ajustado & $\Delta R^{2}$ & $\mathrm{~B}$ & $\mathrm{DE} B$ & $\beta$ & $t$ & $p$ \\
\hline \multicolumn{8}{|l|}{ VD: OCI-R Lavado } \\
\hline & .245 & .103 & & & & & \\
\hline OC-CDQ-Evitación del daño & & & .096 & .035 & .304 & 2.761 & .007 \\
\hline VOCI-just right & & & .086 & .039 & .242 & 2.169 & .032 \\
\hline \multicolumn{8}{|l|}{ VD: OCI-R Comprobación } \\
\hline & .333 & .171 & & & & & \\
\hline VOCI-just right & & & .165 & .048 & .357 & 3.408 & .001 \\
\hline OC-CDQ-Evitación del daño & & & .118 & .043 & .286 & 2.764 & .007 \\
\hline OC-CDQ-Sensación de inacabado & & & -.098 & .049 & -.236 & -2.012 & .047 \\
\hline \multicolumn{8}{|l|}{ VD: OCI-R Orden } \\
\hline & .328 & .150 & & & & & \\
\hline DASS-Ansiedad & & & -.303 & .100 & -.323 & -3.032 & .003 \\
\hline OC-CDQ-Sensación de inacabado & & & .108 & .047 & .272 & 2.313 & .023 \\
\hline NJREQ-R-Número & & & .240 & .109 & .199 & 2.194 & .030 \\
\hline VOCI-just right & & & .099 & .046 & .225 & 2.144 & .034 \\
\hline
\end{tabular}

VD: OCI-R Obsesiones

$.277 \quad .081$

OC-CDQ-Evitación del daño

.103

.041

.274

2.54

.012

VD: OCI-R Acumulación

$.231 \quad .059$

\begin{tabular}{llllll} 
VOCI-Just right & .138 & .053 & .295 & 2.628 & .010 \\
IUS-Intolerancia incertidumbre & .059 & .024 & .325 & 2.488 & .014 \\
\hline
\end{tabular}

VD: OCI-R Neutralización

$.151 \quad .134$

VOCI-just right

.080

.029

.325

2.752

.007

Nota. OCI-R: Obsessive Compulsive Inventory-Revised; DASS: Depression, Anxiety and Stress Scales; OC-CDQ: Obsessive Compulsive Core Dimension Questionnaire; NJREQ-R: Not Just Right Experiences Questionnaire Revised; VD: variable dependiente; VOCI: Vancouver Obsessive Compulsive Inventory; IUS: Intolerance of Uncertainty.

cias not just right y la sensación de inacabado, como la intrusividad y el malestar que generan, ambos fenómenos no son iguales. Las principales diferencias radican en que la sensación de inacabado y las experiencias not just right son más "internas", subjetivas y difusas, y que dichas experiencias tienen lugar cuando el paciente "hace algo", mientras que los contenidos obsesivos se experimentan en muchos casos independientemente de que el paciente lleve a cabo o no una acción determinada.

En cuanto a las relaciones encontradas entre las medidas de la sensación de inacabado, la evitación del daño, el número de experiencias not just right, la gravedad de la experiencia not just right más reciente y los síntomas just right, los resultados mostraron asociaciones moderadas, sugiriendo que los instrumentos empleados miden constructos similares o complementarios, pero no idénticos, puesto que comparten un escaso porcentaje de varianza. Concretamente, se puede observar quela relación más potente se produjo entre la sensación de inacabado y los síntomas just right, lo que indica que estas dos variables mantienen una alta similitud, y difieren en cierta medida de las experiencias not just right (escala de número y gravedad). Este resultado concuerda con el de Ecker y Gönner (2008), quienes en una muestra de pacientes con TOC encontraron una correlación alta $(r=0.70)$ entre la sensación de inacabado y los síntomas just right (cuestionario VOCI). Una de las posi- 
bles explicaciones para esta elevada asociación puede deberse a la semejanza en la redacción de los ítems en los dos instrumentos que se emplean para medir ambos constructos. En sendos casos, los ítems aluden a una tendencia en la forma de pensar, sentir, o actuar de la persona. Por su parte, los ítems que evalúan las experiencias not just right (con el NJRE-Q) están formulados con el objetivo de determinar el número de experiencias not just right que una persona experimenta durante un período temporal concreto (el último mes), y valorar después la gravedad de una experiencia puntual (la experiencia not just right más reciente). Lo que parece indiscutible es que las tres medidas aportan información relevante sobre las motivaciones que, aparentemente, subyacen a los comportamientos de las personas con TOC.

Otro aspecto importante que cabe señalar es la elevada asociación encontrada entre las dimensiones de evitación del daño y la sensación de inacabado. En este sentido, en los modelos de Rasmussen y Eisen (1992) y Summerfeldt et al., (2004), estos constructos se postularon como factores ortogonales y, por tanto, relacionados. De hecho, son varios los estudios que apoyan la validez de la estructura bifactorial, hallando una correlación elevada entre ambas variables (Carrasco y Belloch, 2013; Summerfeldt et al., 2014; Taylor et al., 2014).

Al igual que en otros estudios, se han constatado asociaciones entre las experiencias not just right y la sensación de inacabado con la tendencia al perfeccionismo y la intolerancia a la incertidumbre, así como con las creencias disfuncionales asociadas a los pensamientos. Sin embargo, analizando la magnitud de dichas asociaciones se observa que la evitación del daño y la sensación de inacabado, se asocian significativamente más con la intolerancia a la incertidumbre y con las creencias disfuncionales que con el perfeccionismo. Sin embargo, el número de experiencias not just right, la gravedad de la experiencia not just right más reciente y los síntomas just right, mantienen asociaciones de intensidad similar con la intolerancia a la incertidumbre, el perfeccionismo y las creencias. Este resultado pone en tela de juicio la gran importancia que el perfeccionismo está recibiendo al estudiar las experiencias not just right y la sensación de inacabado, y que se defiende en diversos estudios (p. ej., Coles et al., 2003, 2005; Moretz y McKay, 2009). En cierta medida, esta asociación podría estar justificada, puesto que inicialmente estas experiencias se propusieron como fenómenos que ocurren cuando la persona "busca el estado de perfección". Sin embargo, la clínica nos muestra que las experiencias not just right y la sensación de inacabado no son una mera manifestación del perfeccionismo, ni lo son tampoco en exclusiva del mismo.
En relación a la intolerancia a la incertidumbre, cabe señalar que son pocas las investigaciones que exploran su relación con las experiencias not just right y la sensación de inacabado. Se trata, por lo tanto, de un ámbito todavía muy poco investigado y, teniendo en cuenta los resultados obtenidos en el presente trabajo, parece ser una variable que merece ser analizada en profundidad, especialmente si se tiene en cuenta que la intolerancia a la incertidumbre se postula como una variable transdiagnóstica para un amplio grupo de trastornos emocionales, incluido el TOC (Belloch, 2012).

Las asociaciones mostradas por las variables objeto de estudio con las creencias disfuncionales asociadas a los pensamientos obsesivos son consistentes con los modelos cognitivo-conductuales del TOC (Belloch et al., 2010; Rachman, 2002), que atribuyen a tales creencias un papel mediador entre la experiencia de las intrusiones obsesivas y su conversión en obsesiones clínicas. En este sentido, es de esperar que, puesto que tanto las creencias disfuncionales como las sensaciones de inacabado y las experiencias not just right y síntomas just right se asocian con el TOC, pueden existir a su vez relaciones significativas entre las creencias y dichas sensaciones y experiencias.

Otro importante aspecto está relacionado con el estudio de la posible especificidad de las experiencias not just right, la sensación de inacabado y síntomas just right en la explicación del TOC. Algunos estudios con población general (Coles et al., 2005; Cougle et al., 2011; Pietrefesa y Coles, 2009) y clínica (Sica et al., 2015), mantienen que estos fenómenos son específicos del TOC, situando estas experiencias como hipotéticos marcadores psicológicos del trastorno (Taylor et al., 2014). Sin embargo, Fergus (2013), con una amplia muestra de personas procedentes de la población general, constató que las experiencias not just right eran relevantes para un amplio rango de trastornos emocionales. Es decir, no sólo las personas con TOC se verían afectadas por las experiencias not just right, sino también personas con trastornos diferentes al TOC. Una posible explicación de la presencia de estos fenómenos en un amplio rango de trastornos podría deberse a su vinculación con el afecto negativo, que es una variable transversal y/o transdiagnóstica común a ese mismo rango de trastornos emocionales y afectivos, más que a los síntomas concretos de cada trastorno particular (Aldao, 2012; Sandín, 2012).

Nuestros resultados en relación con la especificidad de estas variables, indican asociaciones moderadas entre las experiencias not just right, la sensación de inacabado, los síntomas just right y la sintomatología emocional TOC y no-TOC, lo que coincide con el estudio de Fergus 
(2013). Aunque la sensación de inacabado y los síntomas just right mostraron asociaciones más potentes con los síntomas obsesivo-compulsivos que con los síntomas no-TOC, también se asociaron con estos últimos. Por su parte, la gravedad de la experiencia not just right más reciente mantuvo correlaciones similares con síntomas obsesivo-compulsivos, de estrés, depresivos, y con la tendencia a preocuparse. Y, finalmente, el número de experiencias not just right se asoció de forma más patente a la sintomatología ansiosa, que al resto de medidas. Estos datos sugieren que la sensación de inacabado y los síntomas just right son, en cierta medida, más específicos de los síntomas TOC que las experiencias not just right.

No obstante, estos resultados deben tomarse con cautela, ya que el estudio de la especificidad de un fenómeno requiere tomar en consideración muestras de pacientes con diversas psicopatologías. Además, cabe la posibilidad de que la inconsistencia de los resultados se deba a un problema en el instrumento de medida de estos fenómenos, o quizás, a la propia definición de especificidad. En cualquier caso, lo que es evidente es que si estos fenómenos se plantean como específicos, se estaría refutando la idea de su "transdiagnosticidad", o de la concepción de estos constructos como endofenotipos, tal y como ha sido planteado por Kloosterman et al., 2013.

Finalmente, los análisis de regresión llevados a cabo indican que las experiencias not just right, la sensación de inacabado y los síntomas just right predicen todas las dimensiones de síntomas obsesivo-compulsivos, por encima del peso explicativo que pueden aportar el perfeccionismo, la intolerancia a la incertidumbre, las creencias disfuncionales, la tendencia a la preocupación patológica (worry), y los síntomas ansiosos y depresivos. Una excepción notable fueron los síntomas de orden, donde la ansiedad fue el predictor más significativo. Este resultado coincide, parcialmente, con el encontrado por Taylor et al., (2014), quienes mostraron que la sensación de inacabado predice los síntomas obsesivo-compulsivos, incluso controlando el peso de la evitación del daño o las creencias disfuncionales relacionadas con los pensamientos. En esta línea, son varios los estudios que encuentran relaciones entre las variables objeto de estudio y las dimensiones de síntomas TOC. En otros trabajos se han encontrado asociaciones entre la sensación de inacabado y la dimensión de orden/simetría (Coles et al., 2003; Ferrão et al., 2012; Pietrefesa y Coles, 2009) y la comprobación (Cougle et al., 2013; Ecker y Gönner, 2008), mientras que otros han encontrado asociaciones entre las experiencias not just right con los síntomas de lavado (Cougle et al., 2011). Con todo, los estudios so- bre las dimensiones de síntomas TOC no son concluyentes, en parte por las limitaciones en cuanto a las medidas y las muestras empleadas.

Este trabajo cuenta con limitaciones que deber ser tenidas en cuenta. Si bien los síntomas que presentan los pacientes con TOC muestran similitudes con ciertas manifestaciones de la población general, los resultados obtenidos en este trabajo deben replicarse en muestras de pacientes con el objetivo de poder generalizar los hallazgos a poblaciones clínicas. Asimismo, la inclusión de un grupo clínico, garantizaría la dimensionalidad de las variables estudiadas. Otra limitación radica en la naturaleza correlacional de los análisis, lo cual impide realizar conclusiones o inferencias causales, así como el empleo exclusivo de medidas de auto-informe.

En conclusión, los resultados derivados de este trabajo, indican que estas "nuevas" variables son relevantes para la explicación del TOC. De hecho, la clínica muestra que algunas de las conductas que llevan a cabo los pacientes con TOC responden a la necesidad de prevenir el daño, pero en muchos otros casos los pacientes se sienten "obligados" a hacerlas, o se sienten "incapaces" de detenerlas, a causa de experiencias o sensaciones internas de que algo no está como debería de estar o ser. Dada la relevancia que estos fenómenos parecen tener, deberían de ser contemplados en los protocolos de evaluación de manera sistemática. Esto es relevante puesto que muchos pacientes no son capaces de expresar estos fenómenos de manera clara, en parte porque no los diferencian de sus síntomas, o porque no los consideran síntomas, y solo a través de la indagación clínica específica, o mediante el uso de instrumentos de auto-informe específicos sería posible ponerlas de manifiesto. Además, sería necesario introducir procedimientos específicos para la disminución o modificación de estas variables en el curso del tratamiento, pues de lo contrario se estaría olvidando un importante componente del trastorno obsesivo compulsivo.

\section{Referencias}

Aldao, A. (2012). Emotion regulation strategies as transdiagnostic processes: A closer look at the invariance of their form and function. Revista de Psicopatología y Psicología Clínica, 17, 261-277.

American Psychiatric Association. (2013). Diagnostic and Statistical Manual of Mental Disorders (5th Edition).Washington, DC: Author.

Arnáez, S., García-Soriano, G., y Belloch, A. (2015). Contenidos obsesivos, miedo a la enfermedad y asco. Revista de Psicopatología y Psicología Clínica, 20, 33-40.

Bados, A., Solanas, A., \& Andrés, R. (2005). Psychometric properties of the Spanish version of Depression, Anxiety and Stress Scales (DASS). Psicothema, 17, 679-683. 
Barajas, S. (2015). Evitación y psicopatología: Un estudio a través de una tarea experimental y su relación con medidas de autoinforme. Revista de Psicopatología y Psicología Clínica, 20, 63-73.

Belloch, A. (2012). Propuestas para un enfoque transdiagnóstico de los trastornos mentales y del comportamiento: Evidencia, utilidad y limitaciones. Revista de Psicopatología y Psicología Clínica, 17, 295-311.

Belloch, A., Morillo, C., Luciano, J. V., García-Soriano, G., Cabedo, E., \& Carrió, C. (2010). Dysfunctional belief domains related to Obsessive-Compulsive Disorder: A further examination of their dimensionality and specificity. The Spanish Journal of Psychology, 13, 369-381.

Belloch, A., Fornés, G., Carrasco, A., López-Solá, C., Alonso, P., \& Menchón, J. M. (2016). Incompleteness and not just right experiences in the explanation of Obsessive-Compulsive Disorder. Psychiatry Research, 236, 1-8.

Belloch, A., Roncero, M., García-Soriano, G., Carrió, C., Cabedo, E. \& Fernández-Álvarez, H. (2013). The Spanish version of the Obsessive-Compulsive Inventory-Revised (OCI-R): Reliability, validity, diagnostic accuracy, and sensitivity to treatment effects in clinical samples. Journal of Obsessive-Compulsive and Related Disorders, 2, 249-256.

Carrasco, A. (2005). Evaluación del perfeccionismo, la indecisión y la intolerancia a la incertidumbre. Trabajo para la obtención del DEA. Universidad de Valencia.

Carrasco, A., y Belloch, A. (2013). Algo no está bien: Una nueva lectura de la duda obsesiva. Psicología Conductual, 21, 341-361.

Carrasco, A., Belloch, A., y Perpiñá, C. (2010). La evaluación del perfeccionismo: Utilidad de la Escala Multidimensional de Perfeccionismo en población española. Análisis y Modificación de Conducta, 36, 49-65.

Coles, M. E., Frost, R. O., Heimberg, R. G., \& Rheaume, J. (2003). "Not just right experiences": Perfectionism, obsessive-compulsive features and general psychopathology. Behaviour Research and Therapy, 41, 681-700.

Coles, M. E, Heimberg, R. G., Frost, R. O., \& Steketee, G. (2005). Not just right experiences and obsessive-compulsive features: Experimental and self-monitoring perspectives. Behaviour Research and Therapy, 43, 153-67.

Cougle, J. R., Fitch, K.E., Jacobson, S., \& Lee, H-J. (2013). A multi-method examination of the role of incompleteness in compulsive checking. Journal of Anxiety Disorders, 27, 231-239.

Cougle, J. R., Goetz, A. R., Fitch, K. E., \& Hawkins, K. A. (2011). Termination of washing compulsions: A problem of internal reference criteria or 'not just right' experience? Journal of Anxiety Disorders, 25, 801-805.

Ecker, W., \& Gönner, S. (2008). Incompleteness and harm avoidance in OCD symptom dimensions. Behaviour Research and Therapy, 46, 895-904.

Ecker, W., Gönner, S., \& Wilm, K. (2011). The measurement of motivational dimensions of OCD: incompleteness and harm avoidance. Psychotherapie, Psychosomatik, Medizinische Psychologie, 61, 62-69.

Fergus, T. A. (2013). Are "Not Just Right Experiences" (NJREs) specific to obsessive-compulsive symptoms?: Evidence that NJREs span across symptoms of emotional disorders. Journal of Clinical Psychology, 0, 1-11.
Ferrão, Y. A., Shavitt, R. G, Prado, H., Fontenelle, L. F., Malavazzi, D. M., de Mathis, M. A., Hounie, A. G., Miguel, E. C., \& do Rosário, M. C. (2012). Sensory phenomena associated with repetitive behaviors in obsessive-compulsive disorder: An exploratory study of 1001 patients. Psychiatry Research, 197, 253-258.

Foa, E. B., Huppert, J. D., Leiberg, S., Langner, R., Kichic, R., Hajcak, G., \& Salkovskis, P. M. (2002). The Obsessive-Compulsive Inventory: Development and validation of a short version. Psychological Assessment, 14, 485-96.

Freeston, M. H., Rhéaume, J., Letarte, H., Dugas, M. J., \& Ladouceur, R. (1994). Why do people worry? Personality and Individual Differences, 17, 791-802.

Frost, R. O., Marten, P., Lahart, C., \& Rosenblate, R. (1990). The Dimensions of Perfectionism. Cognitive Therapy and Research, 14, 449-468.

Janet, P. (1908). Les obsessions et la psychasthénie. Paris: Alcan.

Kloosterman, P. H., Summerfeldt, L. J., Parker, J. D. A, \& Holden, J. J. A. (2013). The obsessive-compulsive trait of Incompleteness in parents of children with autism spectrum disorders. Journal of Obsessive-Compulsive and Related Disorders, 2, 176-182.

Leckman, J. F., Walker, D. E., Goodman, W. K., Pauls, D. L., \& Cohen, D. J. (1994). "Just-Right" perceptions associated with compulsive behaviors in Tourettes's syndrome. American Journal of Psychiatry, 51, 675-680.

Lovibond, P. F., \& Lovibond, S. H. (1995). The structure of negative emotional states: Comparison of the Depression Anxiety Stress Scales (DASS) with the Beck Depression and Anxiety Inventories. Behaviour Research and Therapy, 33, 335-343.

Meyer, T. J., Miller, M. L., Metzger, R. L., \& Borkovec, T. D. (1990). Development and validation of the Penn State Worry Questionnaire. Behaviour Research and Therapy, 28, 487-495.

Miguel, E. C., do Rosário-Campos, M. C., Prado, H. S., do Valle, R., Rauch, S. L., Coffey, B. J., Baer, L., Savage, C. R., O'Sullivan, R. L., Jenike, M. A., \& Leckman, J. F. (2000). Sensory phenomena in obsessive-compulsive disorder and Tourette's disorder. Journal of Clinical Psychiatry, 61, 150-156.

Moretz, M. W., \& McKay, F. D. (2009). The role of perfectionism in obsessive-compulsive symptoms: "Not just right" experiences and checking compulsions. Journal of Anxiety Disorders, 23, 640-644.

Nogueira, R., Godoy, A., Gavino, A., y Romero, P. (2009). Propiedades psicométricas del Vancouver Obsessional Compulsive Inventory (VOCI) en dos muestras no clínicas de población española. Psicothema, 21, 646-651.

Pietrefesa, A., \& Coles, M. E. (2009). Moving beyond an exclusive focus on harm avoidance in Obsessive-Compulsive Disorder: Behavioral validation for the separability of harm avoidance and incompleteness. Behavior Therapy, 40, 251-259.

Rachman, S. (2002). A cognitive theory of compulsive checking. Behaviour Research and Therapy, 40, 625-639.

Rachman, S., \& de Silva, P. (1978). Abnormal and normal obsessions. Behaviour Research and Therapy, 16, 233-248.

Rasmussen, S. A., \& Eisen, J. L. (1992). The epidemiology and clinical features of obsessive compulsive disorder. The Psychiatric Clinics of North America, 15, 743-758

Sandín, B. (2012). Transdiagnostic and clinical psychology: Introduction to the special issue. Revista de Psicopatología y Psicología Clínica, 17, 181-184. 
Sandín, B., Chorot, P., Valiente, R. M., y Lostao, L. (2009). Validación española del cuestionario de preocupación PSWQ: Estructura factorial y propiedades psicométricas. Revista de Psicopatología y Psicología Clínica, 14, 107-122.

Sarmiento, T., Guillen, V., y Sánchez, J. M. (2016). El tratamiento psicológico de la tricotilomanía: un estudio de caso. Revista de Psicopatología y Psicología Clínica, 21, 57-66.

Sica, C., Bottesi, G., Orsucci, A., Pieraccioli, C., Sighinolfi, C., \& Ghisi, M. (2015). "Not Just Right Experiences" are specific to Obsessive-Compulsive Disorder: Further evidence from Italian clinical samples. Journal of Anxiety Disorders, 31, 73-83.

Summerfeldt, L. J. (2004). Understanding and Treating Incompleteness in Obsessive-Compulsive Disorder. Journal of Clinical Psychology, 60, 1155-1168.
Summerfeldt, L. J., Kloosterman, P., Antony, M. M., \& Swinson, R. P. (2014). Examining an obsessive-compulsive core dimensions model: Structural validity of harm avoidance and incompleteness. Journal of Obsessive-Compulsive and Related Disorders, 3, 83-94.

Taylor, S., McKay, D., Abramowitz, J. S, Conelea, C. A., Calamari, J. E., \& Sica, C. (2014). The sense of incompleteness as a motivator of obsessive-compulsive symptoms: An empirical analysis of concepts and correlates. Behavior Therapy, 45, 254-262.

Thordarson, D. S., Radomsky, A. S., Rachman, S., Shafran, R., Sawchuk, C. N., \& Hakstian, A. R. (2004). The Vancouver Obsessional Compulsive Inventory (VOCI). Behaviour Research \& Therapy, 42, 1289-1314. 